\title{
No functional and transductional significance of specific neuropilin 1 siRNA inhibition in colon carcinoma cell lines lacking VEGF receptor 2
}

\author{
MICHAEL QUANTE* , ESTHER RASKOPF* , SEBASTIAN STAHL, ANNABELLE VOGT, \\ TILMAN SAUERBRUCH and VOLKER SCHMITZ
}

Department of Medicine I, University of Bonn, Sigmund-Freud-Strasse 25, 53127 Bonn, Germany

Received July 24, 2008; Accepted November 21, 2008

DOI: 10.3892/or_00000336

\begin{abstract}
Neuropilins are membrane proteins that mediate effects on tumor cells directly and indirectly by affecting angiogenesis. Recent findings indicate that neuropilin 1 (NRP1) and the associated tyrosine kinase vascular endothelial growth factor receptor 2 (VEGFR2) play a regulatory role in developmental angiogenesis as well as in tumor angiogenesis. NRP1 and VEGFR2 might play a role in colon carcinogenesis and development of metastases. The significance of NRP1 expression in colon cancer seems to be controversial. Therefore, we aimed to distinguish between different expression patterns of signalling cascades in human colon carcinoma cell lines in order to analyze the role of NRP1 in tumorigenesis. We analyzed the biological significance of NRP1 in respect to VEGFR, EGFR, neuropilin and their ligands by RT-PCR and Western blot with functional knock-out of NRP1 in different colon adenocarcinoma cell lines. There was no expression of VEGFR2 in tumor cell lines. There were cells that expressed: i) only NRP1 (HT-29, LS174T), ii) NRP2 (Colo320) or iii) both (SW480, LoVo). Cells without NRP1 expression strongly expressed EGFR but only when NRP2 was co-expressed. Inhibition of NRP1 expression by RNA interference did not alter growth characteristics in soft agar experiments. Furthermore, there were no differences in intracellular signalling pathways (ERK1/2 or AKT) in NRP1 inhibited cells. In ex vivo transfer experiments animals with tumors from siRNA-NRP1 transfected cells showed no significant inhibition of tumor growth compared to siRNA control. In conclusion, our results question the role of NRP1 function in VEGFR2 negative colon
\end{abstract}

Correspondence to: Dr Michael Quante, Present address: Division of Digestive and Liver Diseases, Columbia University Medical Center, Irving Cancer Research Center, 1130 St. Nicholas Avenue, New York, NY 10032, USA

E-mail:mq2140@columbia.edu

${ }^{*}$ Contributed equally

Key words: neuropilin, tumorigenesis, siRNA, VEGF, molecular therapy adenocarcinoma cells. NRP1 seems to have no detectable effect on proliferation or migration nor does it induce any changes in intracellular signalling pathways without the expression of VEGFR2. According to our data, further studies are needed to analyze the therapeutic relevance of NRP1 inhibition in vivo.

\section{Introduction}

Colorectal carcinoma is one of the most common malignant diseases in the Western countries. Even after successful resection of the primary tumor, about one-third of the patients develop metastases in the liver and lungs $(1,2)$. Metastatic colorectal cancer is associated with a poor prognosis. Although in many clinical studies chemotherapy is administered in combination with different tyrosine kinase inhibitors $(3,4)$, new approaches for targeted therapies are needed. The antitumoral efficiency of vascular endothelial growth factor (VEGF) antagonism has already been described $(2,5,6)$. VEGF expression correlates with tumor vascularization and therefore tumor growth in colorectal cancer $(5,7,8)$. Treatment with tyrosine kinase inhibitors leads to apoptosis and tumor shrinking. Generally it is accepted that VEGF binds to its cell surface receptors [Flt-1 (VEGFR1), Flk-1 (VEGFR2) or Flt-4 (VEGFR3)] and regulates angiogenesis by either controlling intercellular endothelial interactions or intracellular tumor cell pathways $(8,9)$. In the same way neuropilin (NRP) may indirectly mediate effects on tumor progression by affecting angiogenesis or directly through effects on tumor cells (10).

Neuropilin 1 (NRP1) is a transmembrane glycoprotein with a molecular weight of 130 to $140 \mathrm{kDa}$ that was originally described to be expressed in the developing nervous system (11). Subsequently it was identified as a coreceptor for VEGF suggesting a role in vascularisation and angiogenesis (12). Transgene NRP1 knock-out mice suffer from insufficient and delayed vascularisation leading to embryonic death (13) whereas overexpression is lethal because of hemorrhage and excess blood vessel formation (14). Unlike VEGF receptors, NRP1 does not contain a tyrosine kinase domain and therefore mainly functions as a coreceptor for VEGFR2 for the binding of VEGF165 to induce VEGFR2 related migration and angiogenesis in endothelial cells (15). Several 
Table I. RT-PCR primer pairs.

\begin{tabular}{lllr}
\hline Target & \multicolumn{1}{c}{ Forward primer } & Reverse primer & Fragment \\
\hline VEGFA189 & 5'-GTATAAGTCCTGGAGCGT-3' & 5'-AAACCCTGAGGGAGGCTC-3' & 257 bp \\
VEGFA165/121 & 5'-GTGAATGCAGACCAAAGAAAG-3' & 5'-AAACCCTGAGGGAGGCTC-3' & 100/240 bp \\
VEGFB186/167 & 5'-TGTCCCTGGAAGAACACAGCC-3' & 5'-GCCATGTGTCACCTTCGCA-3' & 260/363 bp \\
VEGFD & 5'-GTATGGACTCTCGCTCAGCAT-3' & 5'-AGGCTCTCTTCATTGCAACAG-3' & 226 bp \\
VEGFR1 & 5'-ATCAGAGATCAGGAAGCACC-3' & 5'-GGAACTTCATCTGGGTCCAT-3' & 452 bp \\
VEGFR2 & 5'-GACTTCAACTGGGAATACCC-3' & 5'-CATGGCCCTGACAAATGTG-3' & 614 bp \\
NRP1 & 5'-AGCCCCTCCTCCTGTTGTGTCCTTC-3' & 5'-GCTATCGCGCTGTCGGTGTAAAAA-3' & 441 bp \\
NRP2 & 5'-CCCCGAACCCAACCAGAAGA-3' & 5'-GAATGCCATCCCAGATGTCCA-3' & 476 bp \\
EGFR & 5'-GGAAGTATGCAGATGC-3' & 5'-CGTAGTGTACGCTTTCG-3' & 239 bp \\
\hline
\end{tabular}

Table II. Real-time RT PCR primer sets.

\begin{tabular}{llll}
\hline Target & \multicolumn{1}{c}{ Forward primer } & \multicolumn{1}{c}{ Reverse primer } & Probe no. \\
\hline Alas & agg cat ggt tcc cag at c & ccc tct tca ccc tgg cta & 64 \\
VEGFA & cac cac ttc gtg atg att ctg & cta cct cca cca tgc caa gt & 63 \\
VEGFB & gct tca cag cac tgt cct & ttt ggg gac atg tcc ctg gaa & 76 \\
VEGFD & tgg tat gaa agg ggc atc tg & tga gtg caa aga aag tct gga & 74 \\
VEGFR1 & cgc ctt acg gaa gct ctc t & cca ctc cct tga aca cga g & 85 \\
NRP1 & cat caa ttt taa ttt ctg ggt tct tt & cac att tca caa gaa gat tgt gc & 85 \\
NRP2 & tgt tgg agc agg gag cat ' & gac ctg gca ctc agg tat cg & 19 \\
EGFR & ggc aca gat gat ttt ggt ca & gat cca agc tgt ccc aat g & 55 \\
\hline
\end{tabular}

studies have further focused on the role of NRP1 in tumor progression and carcinogenesis (11). NRP1 has been associated with increased angiogenesis and tumor growth in vitro and in vivo (16) and is expressed by several nongastrointestinal tumors but also by pancreas, bile duct, gastric and colorectal cancer. In terms of colorectal cancer, it has been reported that NRP1 is expressed in colon adenocarcinoma but not in the adjacent non-malignant colon mucosa (10) and contributes to angiogenesis and suppression of tumor cell apoptosis. Therefore, it was concluded that NRP1 expression may be involved in tumor development with metastasis in colon cancer and may be used as a prognostic factor or therapeutic target $(10,17)$.

Nevertheless, the significance of NRP1 expression in colon cancer has not been clearly characterized to date. Here, we analyze different expression patterns and postreceptor signalling following NRP1 inhibition via RNA interference in different colon cancer cell lines.

\section{Materials and methods}

Animals and cell lines. The tumor cell lines SW480 (ATCC Number: CL228), LoVo(ATCC Number: CCL229), HT-29 (ATCC Number: HTB38), LS174T (ATCC Number: CL188), Colo320 (ATCC Number: CCL220), and Caco2 (ATCC
Number: HTB37) were maintained in DMEM supplemented with $10 \%$ FBS, $200 \mathrm{mM}$ glutamine, $100 \mu \mathrm{g}$ streptomycin, and $100 \mu \mathrm{g}$ penicillin. The human umbilical vein endothelial cell line HUVEC was obtained from Cascade Biologies Inc. (Portland, USA) and cultured in Medium 200 with LSGS. Primary colon epithelial cells were taken from human specimens after routine biopsy.

Eight-week-old male severe combined immunodeficient (SCID) mice were supplied by Charles River (Sulzfeld, Germany) and kept in the local central animal facility of the University Hospital Bonn. The mice were housed under standard conditions and had free access to water and food. Animal procedures were performed in accordance with approved protocols and followed recommendations for proper care and use of laboratory animals.

RT-PCR analysis. All tumor cells, endothelial cells, and primary epithelial cells were analyzed by PCR for expression of VEGFA189, VEGFA165, VEGFA121, VEGFB186, VEGFB 167 and VEGFD, and the receptors VEGFR1, VEGFR2, NRP1, NRP2 and EGFR. Human B-actin was used as positive control. For this, RNA was isolated and reverse transcribed as described previously (18). Sequences are given in Table I. PCR products were separated with agarose gel electrophoresis. 
Table III. Western blot antibodies and conditions.

\begin{tabular}{lccc}
\hline Primary antibody & Protein size $(\mathrm{kDa})$ & Dilution & Secondary antibody \\
\hline EGF (Z-12): sc-275 & 6 & $1 / 200$ & $1 / 2000$ goat anti-rabbit IgG-HRP: sc-2004 \\
EGFR (1005): sc-03 & 170 & $1 / 200$ & $1 / 2000$ goat anti-rabbit IgG-HRP: sc-2004 \\
ERK 1/2 & $44 / 42$ & $1 / 500$ & $1 / 2000$ goat anti-rabbit IgG-HRP: sc-2004 \\
p-ERK1/2 (Thr 177)-R: sc-16981-R & $44 / 42$ & $1 / 500$ & $1 / 1000$ goat anti-mouse IgG-HRP: sc-2005 \\
p-AKT1/2/3 (Ser 473)-R: sc-7985-R & $60 / 56 / 60$ & $1 / 200$ & $1 / 2000$ goat anti-rabbit IgG-HRP: sc-2004 \\
p-p38 (Thr 180/Tyr 182)-R: sc-17852 & 38 & $1 / 200$ & $1 / 2000$ goat anti-rabbit IgG-HRP: sc-2004 \\
PI 3-kinase p85a (B-9): sc-1637 & 85 & $1 / 200$ & $1 / 2000$ goat anti-mouse IgG-HRP: sc-2005 \\
neuropilin (A-12): sc-5307 & 130 & $1 / 200$ & $1 / 2000$ goat anti-mouse IgG-HRP: sc-2005 \\
neuropilin-2 (C-9): sc-13117 & 116 & $1 / 200$ & $1 / 2000$ goat anti-mouse IgG-HRP: sc-2005 \\
Flt-1 (H-225): sc-9029 & 180 & $1 / 200$ & $1 / 2000$ goat anti-rabbit IgG-HRP: sc-2004 \\
Flk-1 (C-20): sc-315 & $150 / 200$ & $1 / 200$ & $1 / 2000$ goat anti-mouse IgG-HRP: sc-2005 \\
B-actin (C4): sc-47778 & 43 & - & $1 / 5000$ goat anti-mouse IgG-HRP: sc-2005 \\
\hline
\end{tabular}

For semiquantitative PCR, RNA was isolated from tumor tissue samples using the HighPure RNA Isolation Tissue Kit (Roche Diagnostics, Mannheim, Germany) according to the manufacturer's protocol. RNA concentrations were determined and RNA was used in the following RT-reaction with random primers (Transcriptor cDNA Synthesis Kit, Roche Diagnostics). Transcript levels of VEGFA189, VEGFA165, VEGFA121, VEGFB186, VEGFB167, and VEGFD and the receptors VEGFR1, VEGFR2, NRP1, NRP2, and EGFR were determined in relation to ALAS mRNA levels by semiquantitative real-time PCR (LightCycler, Roche Diagnostics). Primers were obtained from Invitrogen $\mathrm{GmbH}$ and probes from Roche Diagnostics. Sequences are given in Table II.

Western blot analysis. For analysis of intracellular signal transduction molecules Western blot was performed. Lysates from tumor cells were harvested in a buffer (50 mM Hepes, $\mathrm{pH} 7.4,0.1 \%$ Nonidet P-40, $250 \mathrm{mM} \mathrm{NaCl}$ ) with $1 \mathrm{mM}$ protease and $10 \mathrm{mM}$ phosphatase inhibitors. Whole protein concentrations were determined using the DC protein assay (Bio-Rad, Munich, Germany). Protein was separated by $10 \%$ SDS-PAGE and transferred on nitrocellulose membrane via semi-dry blot. Blocking was performed in 5\% milk and $10 \mathrm{mM}$ Tris $\mathrm{HCl}, \mathrm{pH} 7.4,150 \mathrm{mM} \mathrm{NaCl}, 0.2 \%$ Tween-20 overnight, followed by incubation with primary and secondary antibodies. Detection was done by enhanced chemiluminescence on X-ray film. Primary and secondary antibodies are given in Table III.

Transfection of siRNA. siRNA-NRP1 and a non-sense siRNA (siRNA-CONT) were purchased from Dharmacon (Munich, Germany). The sequences have been selected according to a recent publication (17). A second set of siRNA-NRP1 purchased from Dharmacon as a so-called On-Target plus SMARTpool for NRP1 with defined sequences was used as a control siRNA. Transfection was performed in 6-well plates using 100 pmol siRNA-CONT or siRNA-NRP1 and $5 \mu 1$ Lipofectamine 2000 (Invitrogen) in OptiMEM
(Invitrogen) according to the manufacturer's instructions. Cells were assayed for alterations in expression levels 2 days post transfection after they were ruptured with PBS and three freeze-thaw cycles. Protein concentration was determined using the DC protein assay (Bio-Rad) and $100 \mu \mathrm{g}$ whole protein was analyzed by Western blot. Transfection efficiency was analyzed by transfection of siRNA-GFP (Block-It Fluorescent Oigo, Invitrogen) followed by fluorescence microscopy. Gene knock-down was demonstrated by RT-PCR and Western blot of NRP1 as described above.

Analysis of proliferation after siRNA-treatment. After demonstrating gene knock-down, we tested whether treatment with siRNA-NRP alters functional properties of tumor cells. Selected colon cancer cell lines were transfected with siRNA-NRP or siRNA-CONT as described above and analyzed for changes in proliferation. Proliferation was examined with tetrazolium (MTT); cells were washed with PBS and incubated with MTT at $37^{\circ} \mathrm{C}$ for $45 \mathrm{~min}$. MTT was solubilised with DMSO for 15 min and optical density was determined at $550 \mathrm{~nm}$ (19).

Ex vivo gene transfer of siRNA transfected cells. Orthotopic tumors were established as described previously (19): after transfecting siRNA-NRP and siRNA-CONT in HT-29 and LoVo cells (each $n=5$ ) as described above, $10^{6}$ cells were implanted in the liver of SCID mice $24 \mathrm{~h}$ after transfection (20). Fourteen days after tumor cell inoculation, animals were sacrificed and livers explanted. Tumor growth was determined by measurement of the visible tumor in length and width.

Statistical analysis. Tumor volumes were calculated with the formula length $\mathrm{x}$ width ${ }^{2} \mathrm{x} 0.52$. Data are expressed as mean \pm SEM. Differences of different experimental groups were analyzed for statistical significance by a non-parametric, two-tailed test (Mann-Whitney test) for unpaired samples. Significance was calculated by the log-rank test. $\mathrm{p}<0.05$ was considered to be significant. 


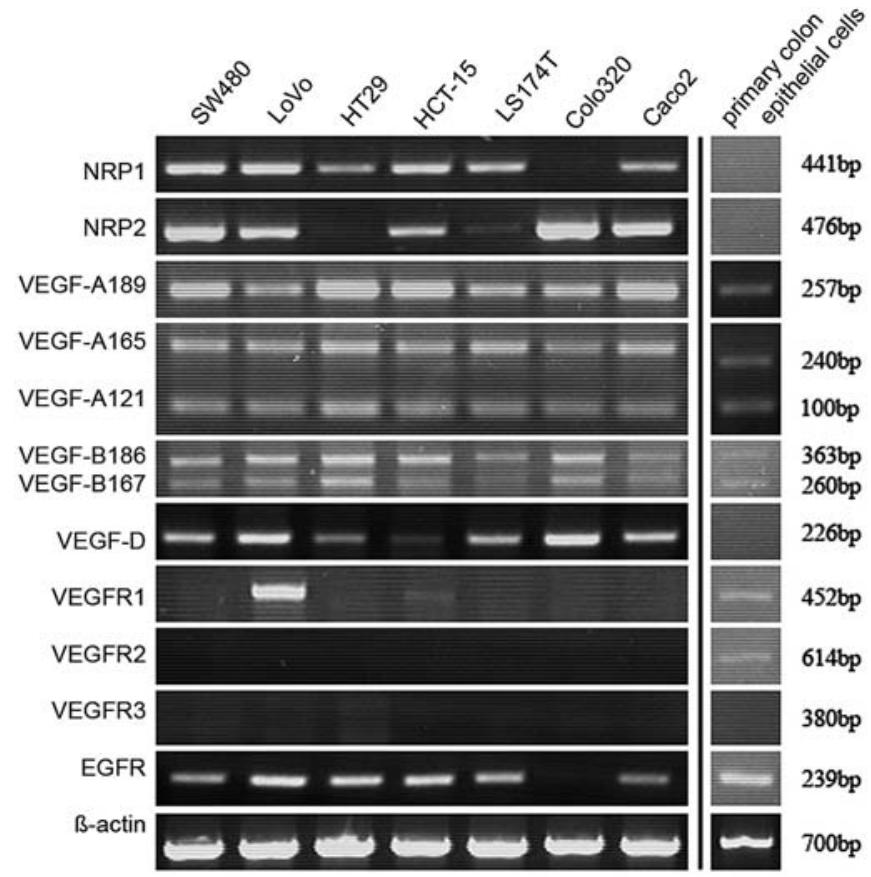

Figure 1. RNA expression profile in different colon cancer cell lines (RT-PCR). RNA from SW480, LoVo, HT-29, HCT-15, LS174T, Colo320, Caco 2 and primary epithelial cells was analyzed by RT-PCR regarding the expression of VEGFA189, VEGFA165, VEGFA121, VEGFB186, VEGFB167 and VEGFD and the receptors VEGFR1, VEGFR2, NRP1, NRP2 and EGFR.

\section{Results}

Receptor expression profile in different colon cancer cell lines. RNA from untreated tumor cells (SW480, LoVo, HT-29, HCT-15, LS174T, Colo320, Caco2) and primary epithelial cells was analyzed by RT-PCR regarding the expression of VEGFA189, VEGFA165, VEGFA121, VEGFB186, VEGFB167, VEGFD and the receptors VEGFR1, VEGFR2, NRP1, NRP2, and EGFR, before starting siRNA-treatment (Fig. 1). All tumor cells and primary epithelial cells expressed VEGFA189, VEGFA165, VEGFA121, VEGFB186, VEGFB167 and VEGFD with slightly different intensity. NRP1 and NRP2 were expressed in all tumor cells except in Colo320, HT-29 and LS174T cells. Both, NRP1 and
NRP2 expression was not detectable in primary epithelial cells. Only in LoVo cells and primary epithelial cells VEGFR1 was expressed. There was no expression of VEGFR2 and VEGFR3 in all analyzed tumor cell lines. Slight expression of VEGFR2 in biopsy samples may possibly be attributable to capillaries and not to epithelium. EGFR was expressed in all, but in Colo320 cells. These results correlated with those of additionally performed real-time RT-PCR experiments (data not shown).

RNA and protein expression profile after inhibition of NRPI. Small interfering RNA transfection in selected tumor cells (SW480, HT-29, LoVo, Colo320) was performed with two different siRNA-NRP1 sequences - one of a recent publication (17) and the other from Dharmacon as a so called OnTarget plus SMARTpool for NRP1 - after optimizing the transfection efficiency up to $90 \%$ (Fig. 2). In tumor cells inhibition of NRP1 expression peaked $48 \mathrm{~h}$ after transfection compared to the control. An additional endothelial cell line (HUVEC) served as a transfection control. Differences in RNA and protein expression pattern (VEGFA189, VEGFA165, VEGFA121, VEGFB186, VEGFB167, VEGFD, VEGFR1, VEGFR2, NRP1, NRP2, EGFR) in tumor cells after treatment with siRNA-NRP1 or siRNA-CONT were analyzed by RT-PCR (Fig. 3) and Western blot (Fig. 4). RNA expression of NRP1 was reduced in SW480, LoVo, HT-29 and in the control cell line (HUVEC) $48 \mathrm{~h}$ after siRNA-NRP1 inhibition. In Colo320 cells NRP1 expression remained undetected. Quantitative analysis of NRP2 expression showed an upregulation of NRP2 in LoVo cells and a downregulation in SW480 and HT-29 cells after siRNA-NRP1 transfection compared to the control. Further quantitative analysis of the associated receptors showed downregulation of EGFR and VEGFR1 in LoVo cells but upregulation of EGFR in HT-29 cells after siRNA-NRP1 transfection compared to the control (Fig. 5). The expression profile of VEGFA189, VEGFA165, VEGFA121, VEGFB186, VEGFB167 and VEGFD remained unchanged after SiRNA-NRP1 transfection compared to the siRNA control.

Protein expression in untreated tumor cells (SW480, LoVo, HT-29, HCT-15, LS174T, Colo320) showed different expression patterns of VEGFR1, VEGFR2, NRP1, NRP2 and EGFR. NRP1 was strongly expressed in HT-29 and LS174T

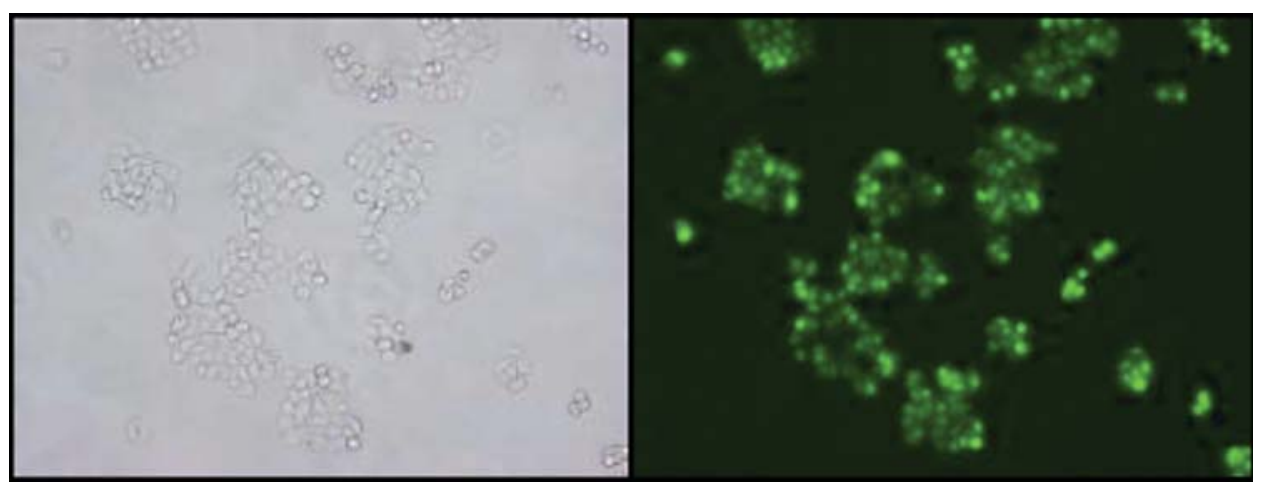

Figure 2. Control of transfection efficiency. siRNA transfection was performed in 6-well plates using Lipofectamine 2000. Transfection efficiency was analyzed by transfection of siRNA-GFP followed by fluorescence microscopy (left, microscopy of in vitro cultured cells; right, fluorescence microscopy of in vitro cultured cells after transfection). 


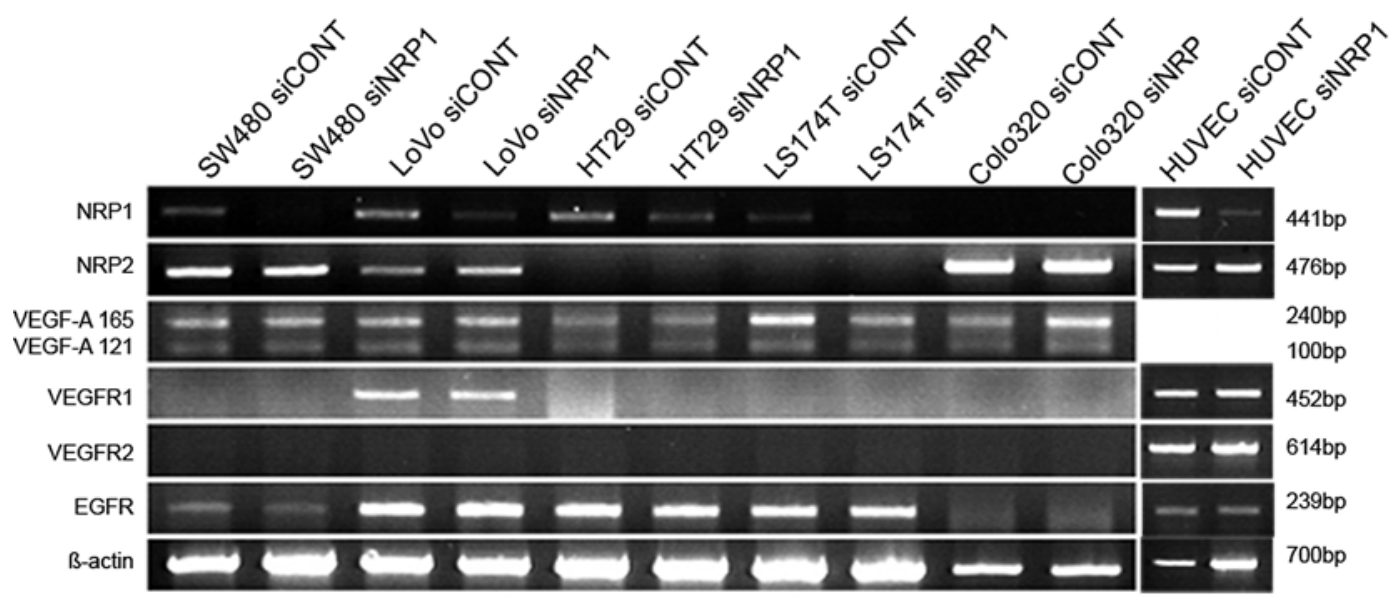

Figure 3. RNA Expression after siRNA transfection. Differences in RNA expression patterns in tumor cells after treatment with siRNA-NRP1 (siNRP1) or control siRNA (siCONT) were analyzed by RT-PCR. Small interfering RNA transfection in selected tumor cells (SW480, HT-29, LoVo, Colo320) was performed with two different siRNA-NRP1 sequences. An additional endothelial cell line (HUVEC) served as a transfection control. RNA expression patterns of VEGFA165, VEGFA121, VEGFR1, VEGFR2, NRP1, NRP2, EGFR correlated with real-time RT PCR.

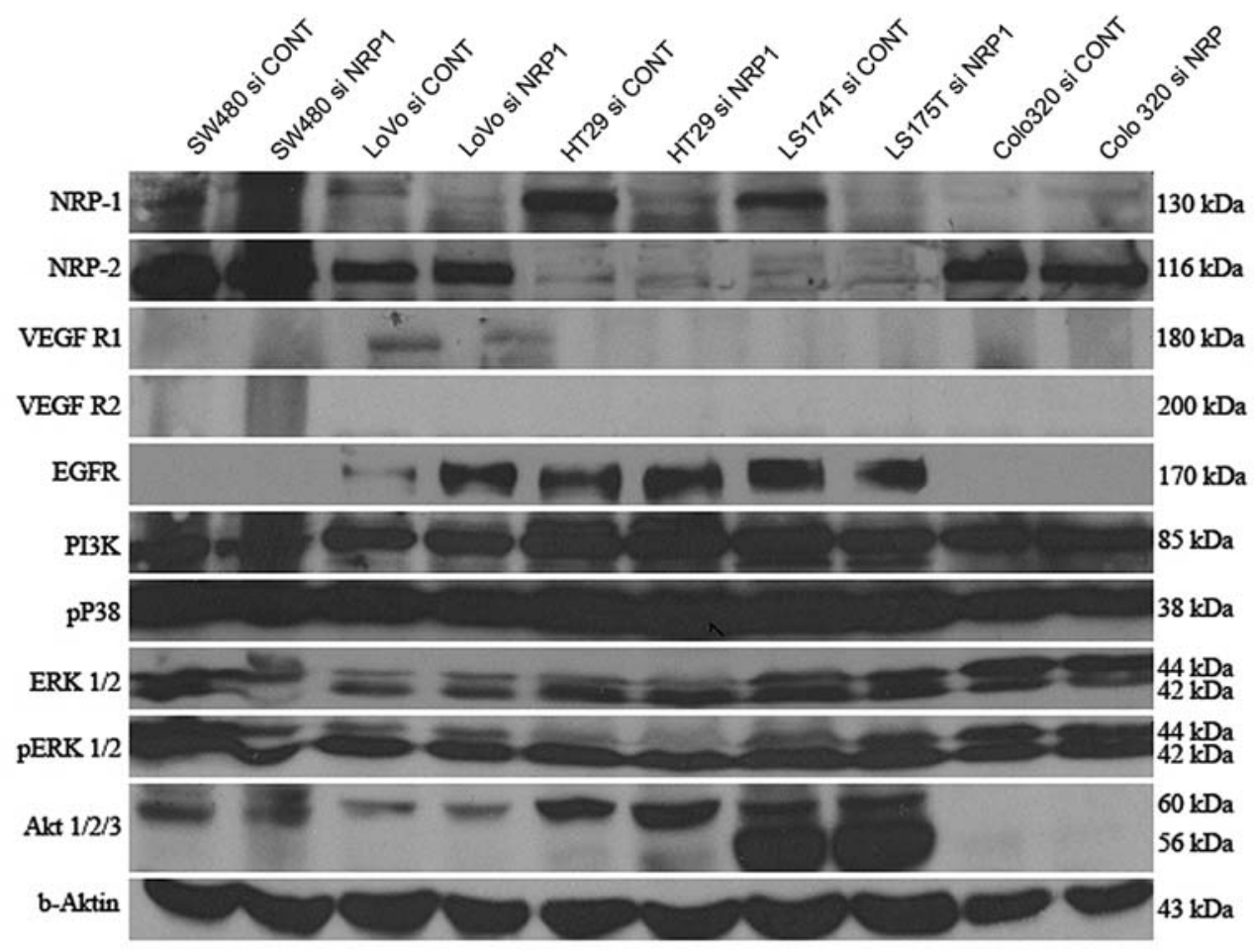

Figure 4. Protein expression after siRNA transfection. Differences in protein expression of receptor and signal cascade proteins in tumor cells after treatment with neuropilin siRNA (siNRP1) or control siRNA (siCONT). siRNA transfection in selected tumor cells (SW480, HT-29, LoVo, LS174T, Colo320) was performed with two different siRNA-NRP1 sequences. For Western blot analysis protein of tumor cells were separated by SDS-PAGE and transferred to nitrocellulose membrane via semi-dry blot, followed by incubation with primary and secondary antibodies (Table III). Detection was done by enhanced chemiluminescence on X-ray film.

and only slightly in SW480 cells. NRP2 was expressed in SW480, LoVo and Colo320 cells. VEGFR1 was only expressed in LoVo cells. VEGFR2 was not expressed in any of the cell lines. EGFR was strongly expressed in HT-29 and LS174T cells and only slightly expressed in LoVo cells (Fig. 4). Transfection of tumor cells with siRNA-NRP1 did not influence protein expression of NRP2 and VEGFR2. NRP1 expression was nearly abolished after siRNA-NRP1 transfection. Furthermore, EGFR expression was induced and VEGFR1 expression reduced after NRP1 transfection in LoVo cells.

Analysis of the effects of NRP inhibition on intracellular signal cascades revealed a strong expression of phosphorylated P38, ERK1/2 and phosphorylated ERK1/2. Phosphorylated AKT1/2/3 was strongly expressed in LS174T followed by HT-29 and SW480 cells and only slightly expressed in Lovo cells. There were no changes in the phosphorylation of AKT1/2/3 or ERK1/2 respectively nor PI3K and p38 MAPK 


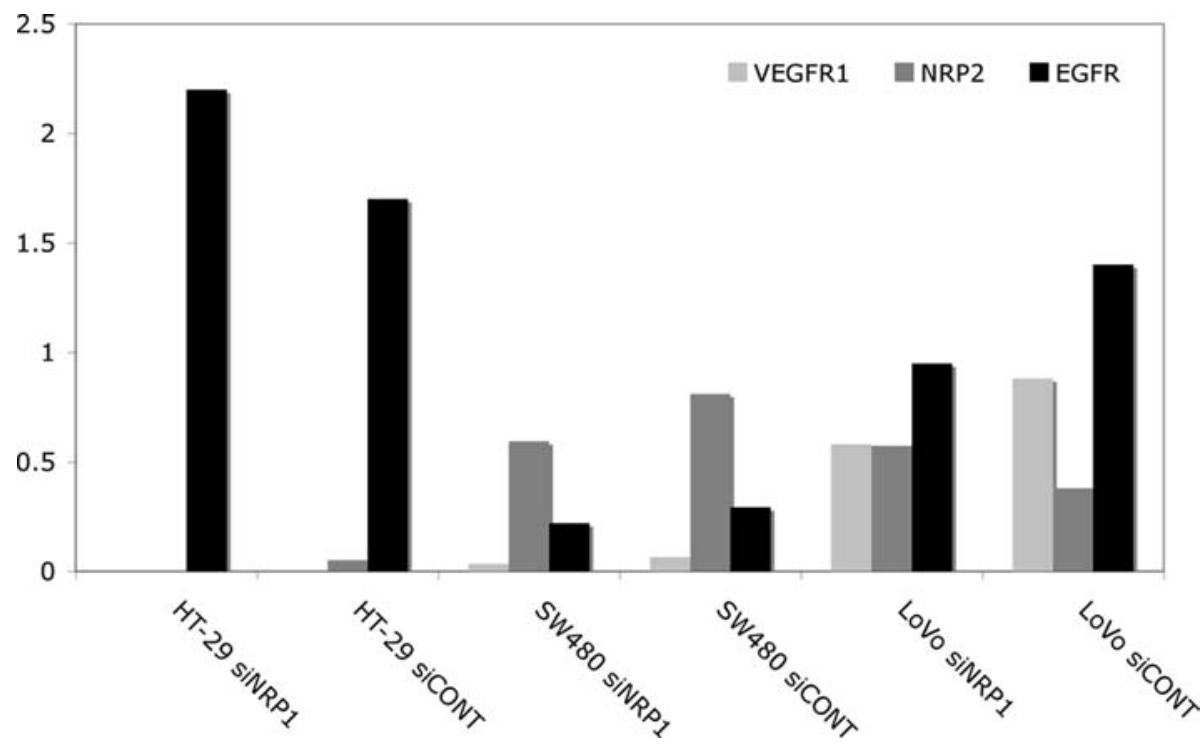

Figure 5. Real-time PCR expression after siRNA transfection. Differences in RNA expression patterns in tumor cells after treatment with siRNA-NRP1 (siNRP1) or control siRNA (siCONT) were analyzed by semiquantitative RT-PCR. Transcript levels of VEGFR1, NRP2, and EGFR were determined in relation to ALAS mRNA levels by semiquantitative real-time PCR (LightCycler). Sequences are given in Table II.

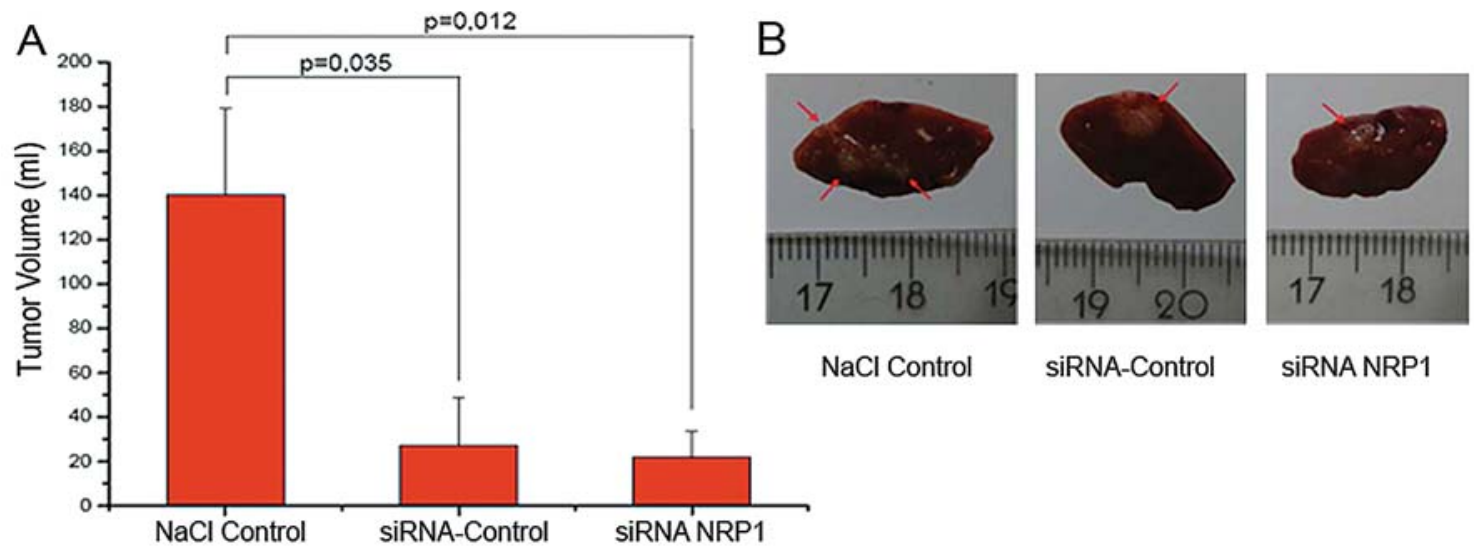

Figure 6. Ex vivo gene transfer were performed in an intrahepatic tumor model. (A) Animals with tumors from siRNA-NRP1 transfected HT-29 and LoVo cells showed no significant inhibition of tumor growth compared to siRNA control. There was a significantly reduced tumor growth of up to $70 \%$ comparing all siRNA treated cells of control and target (NRP1) siRNA to the NaCl treated cells. (B) After transfecting siRNA-NRP and siRNA-CONT in HT-29 and LoVo cells (each $\mathrm{n}=5$ ) as described above, $10^{6}$ cells were implanted in the liver of SCID mice $24 \mathrm{~h}$ after transfection. Fourteen days after tumor cell inoculation, animals were sacrificed and livers explanted.

after siRNA-NRP transfection compared to the control. AKT1/2/3 was not detectable in Colo320 (Fig. 4).

Corresponding to the unchanged effects on signal transduction molecules there was also no effect after siRNANRP1 transfection on proliferation in tumor cells and endothelial cells (data not shown).

Influence of ex vivo transfer of siRNA on orthotopic tumor growth. After verifying the inhibitory effects of siRNA-NRP1 on NRP expression in tumor cell lines, in vivo experiments after ex vivo gene transfer were performed in an intrahepatic tumor model: animals with tumors from siRNA-NRP1 transfected HT-29 and LoVo cells showed no significant inhibition of tumor growth compared to siRNA control (Fig. 6). Interestingly, significantly reduced tumor growth of up to
$70 \%$ was observed comparing all siRNA treated cells of control and target (NRP1) siRNA to the $\mathrm{NaCl}$ treated cells. Both the target and control siRNA could significantly reduce the tumor growth whereas there was no reduction in tumors of $\mathrm{NaCl}$ treated cells.

\section{Discussion}

The members of the neuropilin family were originally identified as receptors for the repulsive axon guidance factor belonging to the class-3 semaphorin sub-family (11). NRP1 is known to function as a coreceptor of VEGFR2 for the binding of VEGF165 and suggested to play a role in embryonic vasculogenesis and angiogenesis $(10,13)$. NRP1 expression has been described as a VEGF coreceptor 
in colon adenocarcinoma and other tumor entities, indicating a role in the process of carcinogenesis by affecting tumor angiogenesis, as well as migration, invasiveness and progression. It was explained that the NRP1 function in tumor cells by tumor-derived NRP1 that acts as a positive modulator of angiogenesis and suggests to target NRP1 as a potential anti-cancer strategy (16). Our results suggest that NRP1 seems to have neither detectable effect on proliferation or migration nor does it activate commonly related intracellular signalling pathways. It does not interact with VEGFR2 in colon cancer cell lines and might interact with VEGFR1 in a metastatic colon cancer cell line (Lovo). Among the analyzed colon cancer cell lines we found cell lines that: i) express NRP1 only (HT-29, LS174T), ii) NRP2 only (Colo320) or iii) both NRP1 and NRP2 (SW480, LoVo). These cell lines represent different stages (Dukes I-III) of colon cancer and further represent a natural knock-out of NRP1 and NRP2.

The fact that there is no VEGFR2 expression in colon adenocarcinoma cell lines corresponds to recent reports from Hansel et al (21) and Parikh et al (10) who did not find VEGFR2 expression in colon cancer. Assuming any functional or transductional significance of NRP1 in VEGFR2 negative colon cancer cell lines these results are contrary to former data indicating an interaction of NRP1 with VEGFR2 to activate cellular proliferation and angiogenesis (12). Pan et al recently reported that blocking of NRP1 and VEGF in tumor models results in an additive effect in reducing tumor growth. But at the same time they present evidence that NRP1 may also be acting through mechanisms other than VEGFR2 signalling. They showed that VEGF121 directly binds to NRP1. However, unlike VEGF165, VEGF121 is not sufficient to bridge the NRP1-VEGFR2 complex. VEGFR2 enhances VEGF165 but not VEGF121 binding to NRP1 $(22,23)$. The lack of downstream effects (except from EGFR upregulation) of VEGF121 or VEGF165 in the absence of VEGFR2 in our study underlines a mandatory role of VEGFR2 for NRP1 signalling. Additionally, this is the first time a correlation of VEGFR1 and NRP1 expression in a metastatic colon cancer cell line has been demonstrated. NRP1 inhibition leads to a downregulation of the tyrosinekinase VEGFR1 in metastatic colon adenocarcinoma cells via an Erk1/2 or Akt-independent pathway. Hansel et al (21) showed that neuropilin is being upregulated during the process of carcinogenesis and correlates to invasive behaviour of gastrointestinal adenocarcinomas. First results from a clinical study from our group (unpublished data) underline the correlation of NRP1 expression and metastases in colon cancer patients with subsequent VEGFR1 upregulation.

The different combination of NRP1 and NRP2 expression in the analyzed cell lines further correlated with a different expression of EGFR. Cells without NRP1 expression did strongly express EGFR and cells with NRP2 expression did not. In metastatic LoVo cells both NRP1 and NRP2 were expressed which resulted in a reduced EGFR expression while VEGFR1 was upregulated. These results suggest an interaction of NRP1 and EGFR although no direct interaction has been proven so far. Recently Wang et al (24) demonstrated that NRP1 induced survival in endothelial cells, which is mediated by a connection of NRP1 with the NRP1 interacting protein. NRP1 activation signals via PI3K/AKT, which subsequently results in an inactivation of $\mathrm{p} 53$ pathways. EGFR acts via the PI3K/AKT pathway. We saw EGFR upregulation when NRP1 was silenced (Fig. 4). This implies an interaction of EGFR and NRP1 independent of PI3K/AKT pathway, which was supported by our data. There is almost no phosphorylated AKT in EGFR-negative cell lines. The expression of NRP2 seems to go along with the lack of EGFR and AKT expression.

To analyze the functional significance of siRNA-NRP1 transfection in selected tumor cells (SW480, HT-29, LoVo, Colo320) was performed. Apart for the described changes of EGFR and VEGFR1 expression in metastatic colon cancer cells, the expected inhibition of NRP1 was observed only. Effects on membrane receptors or signalling pathway proteins were missing. The effect of reduced levels of NRP1 after siRNA-NRP1 transfection on proliferation and apoptosis in tumor cells and endothelial cells did not show any significant differences. Since we used two different siRNA sequences, we can conclude that NRP1 inhibition by siRNA seems to have no effect on tumor cells and therefore is not a sufficient therapy strategy. These findings are controversial to the results of Ochiumi et al (17). Although they did not find any changes in migration ability they demonstrated increased levels of proliferation and angiogenesis with decreased apoptosis in NRP1 knock down cells. The precise mechanism for this inconsistency remains unknown. One explanation might be the different expression of VEGFR2. In the cell lines used in our study, VEGFR2 expression was not detected. Therefore, interaction of NRP1 and VEGFR2 was not found and could not be analyzed.

Although our in vitro data were not in favour of in vivo experiments, previous studies described antitumor efficiency. An intrahepatic tumor model for colon cancer metastasis was chosen and ex vivo gene transfer was performed. Consistent with our in vitro data, siRNA-NRP1 transfected cells showed no significant retardation of tumor growth. Significant reduction of tumor growth of $70 \%$ was observed in all siRNA treated cells (control and NRP1) compared to the $\mathrm{NaCl}$ treated cells. Targeted RNA interference was performed with two different poly U siRNA sequences. Consistently the siRNA itself seems to have an antitumorigenic or apoptotic effect on injected tumor cells. These observations are supported by different reports on non-specific effects of small interfering RNA. These non-specific effects accompanied by proinflammatory responses would be detrimental to a desired therapeutic effect of siRNA. They might be due to an activation of the interferon response and the presence of a 5 '-triphosphate on the RNA $(25,26)$. Judge et al reported that poly U rich siRNA complexes activate the innate and adapted immune system via IFN type I upregulation (27). The NRP1 siRNA sequences employed in our study also contain poly $\mathrm{U}$ rich motif. A current review favors the merging of genesilencing and immune stimulation in one siRNA molecule (28). Nevertheless, inhibition of NRP1 expression with the sequences presented in this study seems to be a controversial experimental tool to analyze the functional effects.

In summary, this report questions the role of NRP1 on carcinogenesis in VEGFR2-negative colon adenocarcinoma 
cells. NRP1 does not seem to have a detectable effect on proliferation or migration nor does it induce any changes in intracellular signalling pathways without the expression of VEGFR2 when analyzing its function by interfering RNA technique. However, in the absence of VEGFR2 there might be an interaction of NRP1 and EGFR. VEGFR1 is upregulated in metastatic colon cancer cells. The relevance of NRP1 inhibition is questionable in this context, despite the fact that the used siRNA itself retarded tumor growth. For any therapeutic approach with NRP1 inhibition by siRNA, further experiments are required to fully understand the interaction of NRP1 and its several coreceptors.

\section{Acknowledgements}

This work was supported by a Deutsche Forschungsgemeinschaft grant to V.S.

\section{References}

1. Ohlsson B and Palsson B: Follow-up after colorectal cancer surgery. Acta Oncol 42: 816-826, 2003.

2. Schmitz V, Kornek M, Hilbert T, et al: Treatment of metastatic colorectal carcinomas by systemic inhibition of vascular endothelial growth factor signaling in mice. World J Gastroenterol 11: 4332-4336, 2005.

3. Willett CG, Duda DG, Czito BG, Bendell JC, Clark JW and Jain RK: Targeted therapy in rectal cancer. Oncology 21: 1055$1065,1075,2007$.

4. Duda DG, Jain RK and Willett CG: Antiangiogenics: the potential role of integrating this novel treatment modality with chemoradiation for solid cancers. J Clin Oncol 25: 4033-4042, 2007.

5. Hanrahan V, Currie MJ, Gunningham SP, et al: The angiogenic switch for vascular endothelial growth factor (VEGF)-A, VEGF-B, VEGF-C, and VEGF-D in the adenoma-carcinoma sequence during colorectal cancer progression. J Pathol 200: 183-194, 2003

6. Schmitz V, Vilanueva H, Raskopf E, et al: Increased VEGF levels induced by anti-VEGF treatment are independent of tumor burden in colorectal carcinomas in mice. Gene Ther 13: 1198-1205, 2006.

7. Fong GH, Rossant J, Gertsenstein M and Breitman ML: Role of the Flt-1 receptor tyrosine kinase in regulating the assembly of vascular endothelium. Nature 376: 66-70, 1995.

8. Takahashi Y, Kitadai Y, Bucana CD, Cleary KR, and Ellis LM: Expression of vascular endothelial growth factor and its receptor, KDR, correlates with vascularity, metastasis, and proliferation of human colon cancer. Cancer Res 55: 3964-3968, 1995.

9. Price DJ, Miralem T, Jiang S, Steinberg R, and Avraham H: Role of vascular endothelial growth factor in the stimulation of cellular invasion and signaling of breast cancer cells. Cell Growth Differ 12: 129-135, 2001.

10. Parikh AA, Fan F, Liu WB, et al: Neuropilin-1 in human colon cancer: expression, regulation, and role in induction of angiogenesis. Am J Pathol 164: 2139-2151, 2004.
11. Guttmann-Raviv N, Kessler O, Shraga-Heled N, Lange T, Herzog Y and Neufeld G: The neuropilins and their role in tumorigenesis and tumor progression. Cancer Lett 231: 1-11, 2006.

12. Soker S, Takashima S, Miao HQ, Neufeld G and Klagsbrun M: Neuropilin-1 is expressed by endothelial and tumor cells as an isoform-specific receptor for vascular endothelial growth factor. Cell 92: 735-745, 1998.

13. Takashima S, Kitakaze M, Asakura M, et al: Targeting of both mouse neuropilin- 1 and neuropilin- 2 genes severely impairs developmental yolk sac and embryonic angiogenesis. Proc Natl Acad Sci USA 99: 3657-3662, 2002

14. Kitsukawa T, Shimono A, Kawakami A, Kondoh $\mathrm{H}$ and Fujisawa H: Overexpression of a membrane protein, neuropilin, in chimeric mice causes anomalies in the cardiovascular system, nervous system and limbs. Development 121: 4309-4318, 1995.

15. Soker S: Neuropilin in the midst of cell migration and retraction. Int J Biochem Cell Biol 33: 433-437, 2001.

16. Miao HQ, Lee P, Lin H, Soker S and Klagsbrun M: Neuropilin-1 expression by tumor cells promotes tumor angiogenesis and progression. FASEB J 14: 2532-2539, 2000.

17. Ochiumi T, Kitadai Y, Tanaka S, Akagi M, Yoshihara M and Chayama K: Neuropilin-1 is involved in regulation of apoptosis and migration of human colon cancer. Int J Oncol 29: 105-116, 2006.

18. Raskopf E, Dzienisowicz C, Hilbert T, et al: Effective angiostatic treatment in a murine metastatic and orthotopic hepatoma model. Hepatology 41: 1233-1240, 2005.

19. Mosmann T: Rapid colorimetric assay for cellular growth and survival: application to proliferation and cytotoxicity assays. J Immunol Methods 65: 55-63, 1983.

20. Schmitz V, Raskopf E, Gonzalez-Carmona MA, et al: Plasminogen fragment K1-5 improves survival in a murine hepatocellular carcinoma model. Gut 56: 271-278, 2007.

21. Hansel DE, Wilentz RE, Yeo CJ, Schulick RD, Montgomery E and Maitra A: Expression of neuropilin-1 in high-grade dysplasia, invasive cancer, and metastases of the human gastrointestinal tract. Am J Surg Pathol 28: 347-356, 2004.

22. Pan Q, Chanthery Y, Liang WC, et al: Blocking neuropilin-1 function has an additive effect with anti-VEGF to inhibit tumor growth. Cancer Cell 11: 53-67, 2007.

23. Pan Q, Chathery Y, Wu Y, et al: Neuropilin-1 binds to VEGF121 and regulates endothelial cell migration and sprouting. J Biol Chem 282: 24049-24056, 2007.

24. Wang L, Dutta SK, Kojima T, et al: Neuropilin-1 modulates p53/caspases axis to promote endothelial cell survival. PLoS ONE 2: e1161, 2007.

25. Kim DH, Longo M, Han Y, Lundberg P, Cantin E and Rossi JJ: Interferon induction by siRNAs and ssRNAs synthesized by phage polymerase. Nat Biotechnol 22: 321-325, 2004.

26. Marques JT, Devosse T, Wang D, et al: A structural basis for discriminating between self and nonself double-stranded RNAs in mammalian cells. Nat Biotechnol 2: 559-565, 2006.

27. Judge AD, Sood V, Shaw JR, Fang D, McClintock K and MacLachlan I: Sequence-dependent stimulation of the mammalian innate immune response by synthetic siRNA. Nat Biotechnol 23: 457-462, 2005.

28. Schlee M, Hornung V and Hartmann G: siRNA and isRNA: two edges of one sword. Mol Ther 14: 463-470, 2006. 